УДК 004.85

\title{
ПРИМЕНЕНИЕ МЕТОДА GLTR ДЛЯ ОПРЕДЕЛЕНИЯ ИСКУССТВЕННЫХ ТЕКСТОВ НА РУССКОМ ЯЗЫКЕ
}

\author{
Конопаткин Олег Владимирович \\ магистрант \\ Научный руководитель: Добров Борис Викторович \\ к. ф.-м. н., зав. лаб. НИВЦ МГУ \\ ФГБОУ ВО «МГУ имени М. В. Ломоносова»
}

\begin{abstract}
Аннотация: современные нейронные сети на основе архитектуры Transformer, такие как GPT и BERT, позволяют генерировать искусственные тексты, на первый взгляд не отличимые от написанных человеком. В данной работе исследуется возможность применения метода GLTR, созданного для определения искусственных текстов, сгенерированных именно такими нейронными сетями, к русскоязычным текстам.

Ключевые слова: GLTR, машинное обучение, классификация текстов, GPT, BERT.
\end{abstract}

\section{APPL YING GLTR FOR ARTIFICIAL RUSSIAN TEXTS DETECTION}

Konopatkin Oleg Vladimirovich

Scientific adviser: Dobrov Boris Viktorovich

\begin{abstract}
Transformer architecture, such as GPT of BERT, can generate artificial texts indistinguishable from humanwritten texts at first glance. This work explores possibility of applying GLTR method, created specifically for detecting artificial texts generated by such neural networks, to russian texts.
\end{abstract}

Key words: GLTR, machine learning, text classification, GPT, BERT.

\section{Данные}

Для проведения исследования были загружены данные открытого корпуса текстов на русском языке Taiga[1], на основе которых затем была произведена генерация искусственных текстов, для генерации использовались тексты из новостного подкорпуса и открытая предобученная сеть ruGPT3 от 


\section{ВСЕРОССИЙСКИЙ ИССЛЕДОВАТЕЛЬСКИЙ ФОРУМ

лаборатории sberbank-AI[2]. Генерация проводилась при помощи top-k/top-p сэмплирования.

В результате генерации были получены следующие наборы искусственных текстов:

- 50000 текстов для $\mathrm{k}=0, \mathrm{p}=1.0$.

- 35000 текстов для $\mathrm{k}=20, \mathrm{p}=0.95$.

- 50000 текстов для $\mathrm{k}=5, \mathrm{p}=0.95$.

Длина сгенерированных текстов была ограничена 100 словами, генерация проводилась как продолжение префиксов случайной длины (15-25 слов) текстов из загруженной коллекции. Из исходных и сгенерированных текстов были собраны обучающие коллекции с различными пропорциями исходных и искусственных текстов. Для исключения влияния длины текста на классификацию, исходные тексты также были ограничены по длине 100 словами. В связи с избыточным для данной работы размером корпуса Taiga, исходные новостные тексты были взяты из сегмента новостей с сайта fontanka.ru, и эти же тексты использовались в качестве положительного класса в составе собранных обучающих коллекций.

Экспериментальные коллекции были сформированы из выбранных сгенерированных текстов и естественных текстов, префиксы которых использовались для генерации (табл. 1). В таблице перечислены все сформированные коллекции и их характеристики. В составе коллекции описано, сколько текстов из каждой группы (естественные или сгенерированные с заданными параметрами) содержится в коллекции. Размеры обучающей и тестовой выборки показывают только количество текстов в них, поскольку разбиение проводилось с сохранением доли текстов из каждой группы в обеих выборках.

Таблица 1

\section{Характеристики экспериментальных коллекций}

\begin{tabular}{|l|l|l|l|}
\hline \multicolumn{1}{|c|}{ Идентификатор } & \multicolumn{1}{|c|}{ Состав коллекции } & \multicolumn{1}{|c|}{$\begin{array}{c}\text { Размер обучающей } \\
\text { выборки }\end{array}$} & $\begin{array}{c}\text { Размер тестовой } \\
\text { выборки }\end{array}$ \\
\hline A_5/0.95 & $\begin{array}{l}50000 \times \text { (natural) } \\
50000 \times(\mathrm{k}=5, \mathrm{p}=0.95)\end{array}$ & 80000 & 20000 \\
\hline A_5/0.95_small & $\begin{array}{l}7500 \times(\text { natural }) \\
7500 \times(\mathrm{k}=5, \mathrm{p}=0.95)\end{array}$ & 14000 & 1000 \\
\hline B_5/0.95 & $\begin{array}{l}25000 \times(\text { natural }) \\
25000 \times(\mathrm{k}=5, \mathrm{p}=0.95)\end{array}$ & 40000 & 10000 \\
\hline
\end{tabular}




\begin{tabular}{|c|c|c|c|}
\hline Идентификатор & Состав коллекции & $\begin{array}{c}\text { Размер обучающей } \\
\text { выборки }\end{array}$ & $\begin{array}{c}\text { Размер тестовой } \\
\text { выборки }\end{array}$ \\
\hline B_20/0.95 & $\begin{array}{l}25000 \times(\text { natural }) \\
25000 \times(\mathrm{k}=20, \mathrm{p}=0.95)\end{array}$ & 40000 & 10000 \\
\hline B_0/1.0 & $\begin{array}{l}25000 \times \text { (natural) } \\
25000 \times(\mathrm{k}=0, \mathrm{p}=1.0)\end{array}$ & 40000 & 10000 \\
\hline C_25/10/10/10 & $\begin{array}{l}25000 \times(\text { natural }) \\
10000 \times(\mathrm{k}=5, \mathrm{p}=0.95) \\
10000 \times(\mathrm{k}=20, \mathrm{p}=0.95) \\
10000 \times(\mathrm{k}=0, \mathrm{p}=1.0)\end{array}$ & 44000 & 11000 \\
\hline C_20/20/20/20 & $\begin{array}{l}20000 \times(\text { natural }) \\
20000 \times(\mathrm{k}=5, \mathrm{p}=0.95) \\
20000 \times(\mathrm{k}=20, \mathrm{p}=0.95) \\
20000 \times(\mathrm{k}=0, \mathrm{p}=1.0)\end{array}$ & 64000 & 16000 \\
\hline
\end{tabular}

Сформированные коллекции разбиваются на 3 группы: группа А для предварительной оценки рассмотренных методов, группа В для исследования влияния параметров генерации искусственных текстов на качество классификации, и группа С для исследования влияния наличия в обучающей коллекции искусственных текстов разных типов.

\section{Использованные методы}

В ходе экспериментов были использованы две группы методов машинного обучения. Первая группа - стандартные методы на представлении текстов мешком слов с tf-idf мерой, а именно:

- Логистическая регрессия

- Метод опорных векторов

- Наивный метод Байеса

- Метод ближайших соседей/центроидов

- Деревья решений, случайный лес

- Адаптивный бустинг

Вторая группа - методы на основе нейронных сетей, к которым относятся классификатор на основе предобученной сети ruBERT из библиотеки deeppavlov[3] и метод GLTR[4]. Метод GLTR сам по себе не является классификатором, a только выделяет признаки из входного текста с использованием нейронной сети архитектуры BERT или GPT. Для каждого слова входного текста вычисляются значения top_k, frac(p) и top_10_entropy(p) [4, с. 2], предназначенные для обнаружения особенностей искусственно сгенерированных моделями на основе архитектуры Transformer текстов. 


\section{ВСЕРОССИЙСКИЙ ИССЛЕДОВАТЕЛЬСКИЙ ФОРУМ

\section{Эксперименты}

Для предварительного сравнения качества работы рассмотренных методов, они были применены к тестовым коллекциям группы А, полученные результаты приведены в таблице (табл. 2).

Использование GLTR на основе порождавшей тексты модели даёт наилучшую аккуратность классификации (классификатор на основе метода опорных векторов по выделенным GLTR признакам). Следует отметить, что аккуратность классификации сильно падает при использовании GLTR на основе ruBERT и становится меньше, чем для стандартных методов. Для англоязычных текстов эта разница сильно меньше (не более 5\% в худшем случае [4, с. 4]). Такой результат, возможно, объясняется тем, что модели, обученные на англоязычных текстах, выучивают более похожие внутренние представления языковой модели и предсказывают более схожие вероятности появления слов в заданном контексте, поскольку порядок слов в английском языке фиксирован более жёстко, чем в русском.

Таблица 2

\section{Результаты классификации коллекций группы А}

\begin{tabular}{|l|l|l|l|}
\hline Группа методов & Наибольшая аккуратность & Наилучший алгоритм & F-мера \\
\hline Стандартные методы & 0.876 & Ridge Classifier & 0.875 \\
\hline ruBERT & 0.936 & ruBERT & 0.935 \\
\hline GLTR(ruGPT-3) & $\mathbf{0 . 9 6 0}$ & SVC & $\mathbf{0 . 9 6 1}$ \\
\hline GLTR(ruBERT) & 0.803 & Random Forest & 0.798 \\
\hline
\end{tabular}

В таблице (табл. 3) приведены результаты применения рассмотренных методов к коллекциям из групп В и С. Лучшего результата во всех случаях достигает метод GLTR на основе ruGPT-3, поэтому в таблице приведены только лучшие методы на полученных им признаках.

Таблица 3

\section{Результаты классификации коллекций группы В}

\begin{tabular}{|l|l|l|l|}
\hline Коллекция & Наибольшая аккуратность & Наилучший алгоритм & F-мера \\
\hline B_5/0.95 & 0.9538 & KNeighbors Classifier & 0.9544 \\
\hline B_20/0.95 & 0.9325 & RandomForest Classifier & 0.9314 \\
\hline B_0/1.0 & 0.8627 & AdaBoost Classifier & 0.8606 \\
\hline C_25/10/10/10 & 0.8737 & AdaBoost Classifier & 0.8600 \\
\hline C_20/20/20/20 & 0.8944 & AdaBoost Classifier & 0.7632 \\
\hline
\end{tabular}




\section{ВСЕРОССИЙСКИЙ ИССЛЕДОВАТЕЛЬСКИЙ ФОРУМ

Эти результаты показывают, что метод GLTR лучше всего позволяет классифицировать искусственные тексты, сгенерированные с сильными ограничениями top-k и top-p. При этом для коллекций со смешанными типами сгенерированных текстов показано качество ближе к наиболее сложной из групп текстов, представленных в смешанной коллекции.

\section{Дополнительные эксперименты}

Для проверки обобщаемости полученного классификатора на основе GLTR был проведён ряд дополнительных экспериментов:

1) Тестирование оптимального классификатора на искусственных текстах, сгенерированных на Яндекс.Рефератах[5]. Из 100 текстов только один был классифицирован как искусственный.

2) Тестирование оптимального классификатора на текстах, сгенерированных сетью ruGPT-3 на более коротких префиксах естественных текстов. Наихудший результат показало тестирование для префиксов длиной 5 слов (54.8\%), а наилучший - для префиксов длиной 25 слов (91.6\%).

3) Тестирование оптимального классификатора только на самых коротких текстах тестовой выборки. Аккуратность на текстах, длиной короче 30 слов оказалась на $22.7 \%$ ниже, чем на всей тестовой выборке (69.7\% против $92.4 \%)$.

Полученные результаты показывают, что метод GLTR заметно переобучается под данные обучающей выборки.

\section{Заключение}

В ходе данной работы было продемонстрировано, что метод GLTR на основе модели ruGPT-3 показывает наилучшее среди рассмотренных методов качество на искусственных текстах, сгенерированных этой же моделью. Тем не менее, также было продемонстрировано, что этот метод имеет тенденцию к переобучению на данных из обучающей выборки. В качестве частичного решения этой проблемы предлагается включать более разнообразные типы искусственно сгенерированных текстов в обучающую выборку при использовании методов машинного обучения на GLTR признаках.

\section{Список литературы}

1. Taiga Corpus [Электронный pecypc]. - Электрон. дан. - URL: https://tatianashavrina.github.io/taiga_site/downloads (дата обращения: 18.04.2021). 
2. Sberbank AI ruGPTs [Электронный ресурс]. - Электрон. дан. - URL: https://github.com/sberbank-ai/ru-gpts (дата обращения: 18.04.2021).

3. DeepPavlov BERT models [Электронный pecypc]. - Электрон. дан. URL: http://docs.deeppavlov.ai/en/master/features/models/bert.html (дата обращения: 18.04.2021).

4. Sebastian Gehrmann, Hendrik Strobelt, Alexander M. Rush. GLTR: Statistical Detection and Visualization of Generated Text. // NIPS, 2019. - 6 p.

(C) О.В. Конопаткин, 2021 